\title{
Analysis of codon usage pattern in Taenia saginata based on a transcriptome dataset
}

\author{
Xing Yang ${ }^{1,2}$, Xuenong Luo ${ }^{1}$ and Xuepeng $\mathrm{Cai}^{1,2^{*}}$
}

\begin{abstract}
Background: Codon usage bias is an important evolutionary feature in a genome and has been widely documented in many genomes. Analysis of codon usage bias has significance for mRNA translation, design of transgenes, new gene discovery, and studies of molecular biology and evolution, etc. However, the information about synonymous codon usage pattern of $T$. saginata genome remains unclear. T. saginata is a food-borne zoonotic cestode which infects approximataely 50 million humans worldwide, and causes significant health problems to the host and considerable socio-economic losses as a consequence. In this study, synonymous codon usage in $T$. saginata were examined.

Methods: Total RNA was isolated from T. saginata cysticerci and 91,487 unigenes were generated using Illumina sequencing technology. After filtering, the final sequence collection containing 11,399 CDSs was used for our analysis.

Results: Neutrality analysis showed that the T. saginata had a wide GC3 distribution and a significant correlation was observed between GC12 and GC3. NC-plot showed most of genes on or close to the expected curve, but only a few points with low-ENC values were below it, suggesting that mutational bias plays a major role in shaping codon usage. The Parity Rule 2 plot (PR2) analysis showed that GC and AT were not used proportionally. We also identified twenty-three optimal codons in the T. saginata genome, all of which were ended with a $\mathrm{G}$ or $\mathrm{C}$ residue. These results suggest that mutational and selection forces are probably driving factors of codon usage bias in T. saginata genome. Meanwhile, other factors such as protein length, gene expression, GC content of genes, the hydropathicity of each protein also influence codon usage.

Conclusions: Here, we systematically analyzed the codon usage pattern and identified factors shaping in codon usage bias in T. saginata. Currently, no complete nuclear genome is available for codon usage analysis at the genome level in T. saginata. This is the first report to investigate codon biology in T. sagninata. Such information does not only bring about a new perspective for understanding the mechanisms of biased usage of synonymous codons but also provide useful clues for molecular genetic engineering and evolutionary studies.
\end{abstract}

Keywords: Taenia saginata, Codon usage bias, Trancriptome, Optimal codon

\section{Background}

Codon usage bias (CUB) refers to the phenomenon where synonymous codons are not used with equal frequencies during translation of genes. CUB is a common phenomenon in a wide variety of organisms, including prokaryotes and eukaryotes [1-3]. Many factors have been reported to influence codon usage in various organisms. Weak natural selection and mutational pressure are thought to be the

\footnotetext{
*Correspondence: caixp@vip.163.com

'State Key Laboratory of Veterinary Etiological Biology, Lanzhou Veterinary Research Institute, Chinese Academy of Agricultural Sciences, Lanzhou 730046, PR China

${ }^{2}$ College of Veterinary Medicine, Jilin University, Changchun 130000, PR China
}

\section{Biomed Central}

main factors that account for the codon usage variation among the genes in these organisms [4]. Genome-wide investigations of codon usage patterns has an immense importance in understanding the basic features of molecular organization of a genome. In addition, analysis of CUB has many other important applied aspects, such as heterologous gene expression [5], the determining of the origins of species [6], the design of degenerate primers [7], the prediction of expression level of genes [8,9], as well as the prediction of gene functions [10]. However, most of numerous reports on CUB have focused on model organisms and many microorganisms, such as Caenorhabditis, Drosophila, Arabidopsis [11], yeast [12], Giardia lamblia [13], 
Entamoeba histolytica [14], Streptomyces [15], Borrelia burgdorferi [16], and Saccharomyces cerevisiae [17]. For example, in C. elegans it is observed that most favored codons are ended with $\mathrm{G}$ and/or $\mathrm{C}$ (majority are $\mathrm{C}$ ending) [18]. In contrast, there are few studies on tapeworms. $T$. saginata is an important parasitic tapeworm which is widely distributed in the world [19]. The adult worms mainly parasitize in the small intestines of humans [20,21]. T. saginata can cause great economic losses and endangers public health [22,23]. However, the information about synonymous codon usage pattern of $T$. saginata remains unclear. In this study, we investigated the codon usage profile of $T$. saginata through transcriptome data using a multivariate statistical analysis. Analysis of codon usage pattern in T. saginata would provide a basis for understanding the related mechanism for biased usage of synonymous codons and for choosing appropriate host expression systems for an optimized expression of target genes.

\section{Methods}

\section{Ethics statement}

This study was approved by the Animal Ethics Committee of Lanzhou Veterinary Research Institute, Chinese Academy of Agricultural Sciences (Approval No. LVRIAEC2009-2012). The cattle from which Taenia saginata cysticerci were collected for transcriptome sequencing, were handled in accordance with good animal practices required by the Animal Ethics Procedures and Guidelines of the People's Republic of China.

\section{RNA extraction, cDNA library preparation and Illumina sequencing}

Total RNA was extracted from cysticerci using the Trizol reagent (Invitrogen, Carlsbad,CA), following the manufacturers instructions. The quantity and quality of total RNA was analyzed using Agilent 2100 RNA Nanochip (Agilent, Santa Clara, CA, USA) and gel electrophoresis. A total of $16.1 \mu \mathrm{g}$ of RNA was pooled for the preparation of the cDNA library.

The OligoTex mRNA mini kit (Qiagen) was used to poly-T+ RNA after total RNA was collected according to the manufacturer's protocol. The mRNA was mixed with fragmentation buffer and fragmented into short fragments. cDNA was synthesized using the mRNA fragments as templates. Short fragment ( $200 \pm 25 \mathrm{bp})$ were gel extracted from an agarose gel and PCR amplified for 15 cycles. Finally, the library was sequenced using the Illumina HiSeq 2000 sequencer (Beijing Genomics Institute, BGI, Shenzhen, Guangdong, China).

\section{De novo assembly}

Using Solexa/Illumina RNA-seq deep sequencing technology, we obtained a total of 55.49 million raw reads (4.99 Gb). Further, raw reads were filtered to remove the low-quality reads. The filtration steps were as follows: 1 ) remove adaptor sequence; 2 ) remove reads containing the unknown nucleotide "N" over 10\%; 3) remove low quality reads containing more than 10 bases with Q-value $\leq 20$. Then, the remaining high-quality reads were used for further analysis. Transcriptome raw reads dataset has been submitted to the NCBI (http://www.ncbi.nlm.nih.gov/ bioproject/PRJNA260140).

\section{Sequence data}

In this study, a total of 91,487 T. saginata unigenes were obtained. Based on a sequence similarity with known proteins, a total of 59,262 unigenes were annotated. Up to 57,607 of which were annotated against the NCBI nonredundant $(\mathrm{Nr})$ protein database, 24,860 were assigned to the protein database Clusters of Orthologous Groups (COG), 26,476 were assigned to the term annotation database of Gene Ontology (GO), and 43,575 were assigned to 200 pathways in the database of Kyoto Encyclopedia of Genes and Genomes (KEGG). Among the annotated unigenes, 61,941 coding sequences (CDS) were obtained by the BLASTx algorithm [24]. All CDSs were analyzed using the FrameDP software [25], which has the ability to selftrain directly on EST clusters instead of requiring curated cDNA sets to train the underlying ESTScan and DECODER software [26].

To minimise the sampling error, only CDS sequences longer than $300 \mathrm{bp}$ were used for this study. The final sequence collection containing 11,399 CDSs was used for our analyses.

\section{Indices of codon usage}

Codon usage in these genes was assessed using the program codonW 1.4.4 (J Peden, http://codonw.sourceforge. net). Relative synonymous codon usage (RSCU) is the observed frequency of a codon divided by the frequency expected, if all synonyms for that amino acid were used equally [27]. Thus, RSCU values close to 1.0 indicate lack of bias whereas values more than 1 indicates that a codon was used more frequently than expected, while the converse is true for RSCU values less than 1. The effective number of codons (ENC) method was used to quantify the absolute codon usage bias of a CDS [28]. The values of ENC range from 20 (when only one codon is used per amino acid) to 61 (when all codons are used in equal probability). The predicted values of ENC were calculated as

$$
E N C=2+s+\frac{29}{s^{2}+\left(1-s^{2}\right)}
$$

where s represents the given $(\mathrm{G}+\mathrm{C})_{3} \%$ value [28].

To determine the preferred codon for each synonymous codon group, the 'relative synonymous codon usage' 
RSCU values were calculated according to the formula of previous reports [27].

$$
R S C U=\frac{g i j}{\sum_{j}^{n i} g i j} n j
$$

where gij is the observed number of the $i$ th codon for $j$ th amino acid which has $n_{i}$ type of synonymous codons. The codon with RSCU value more than 1.0 has positive codon usage bias, while the value $<1.0$ has relative negative codon usage bias. When RSCU value is equal to 1.0, it means that this codon is chosen equally and randomly.

The GC content of first, second and third codon position (GC1, GC2 and GC3 respectively) were then calculated. GC12 is the average of GC 1 and GC2, and was used for analysis of neutrality plots (GC12vsGC3) [29]. The codon adaptation index (CAI) was used to estimate the extent of bias toward codons that were known to be preferred in highly expressed genes. A CAI value is between 0 and 1.0, and a higher value means a likely stronger codon usage bias and a potential higher expression level [30].

\section{Correspondence analysis(CA)}

Correspondence analysis (CA) has been widely used to explore codon usage variation among genes. CA is a sophisticated multivariate statistical technique in which the codon usage data (59 codons) are plotted in a multidimensional space of 59 axes (excluding Met, Trp and stop codons) and then it identifies the axes which represent the most prominent factors contributing to variation among genes $[31,32]$.

\section{Determination of optimal codons}

We selected $5 \%$ of the total genes with extremely high and low CAI values which were regarded as the high and low expression genes datasets, respectively. Codon usage was compared using Chi squared contingency test of the two groups, and codons whose frequency of usage were significantly higher $(\mathrm{P}<0.01)$ in highly expressed genes than in genes with low level of expression would be defined as the optimal codons [33].

\section{Statistical analysis}

CodonW 1.4.4 software was used to analyze the indices of codon usage. Correlation analysis was carried out using the Spearman's rank correlation analysis method wrapped in the multianalysis software SPSS version 19.0.

\section{Results}

\section{Codon usage in $T$. saginata}

The pattern of synonymous codon usage in the T. saginata has been shown in Table 1 . The genomic $\mathrm{G}+\mathrm{C}$ content for T. saginata is $43.61 \%$. Although the genome would thus appear to be slightly $\mathrm{A}+\mathrm{T}$ rich, overall codon usage is biased toward C- and G-ending codons (32 codons were frequently used codons, 18/32 of the frequently used codons end with $\mathrm{G}$ or $\mathrm{C}$ ), suggesting the compositional constraints are not the most important factor in shaping the codon usage variation among the genes.

In general, the pattern of codon usage is similar among closely related organisms, but differs significantly among distantly related species, such as Escherichia coli, Saccharomyces cerevisiae and Drosophila melanogaster [34]. In this study, patterns of codon usage are compared in $T$. saginata and T. pisiformis (Table 1) [35], and we found that there are high similarities between them. With the exception of UCA and GGA, the two species have the same preferred codon for all amino acids.

\section{Nucleotide content of genes}

The GC content of the $T$. saginata genes varied from $31 \%$ to $80.2 \%$ with a standard deviation(SD) of 3.67 . The GC content of 11399 genes were mainly distributed between $45 \%$ and $55 \%$ (Figure 1), this distribution pattern of genes is very similar to T. pisiformis [35]. To understand the nucleotide distribution, we concatenated all genes to one sequence, which comprised 532,4389 codons. The GC content in 3 codon positions (GC1, GC2, and GC3) was $0.534,0.439$, and 0.535 , respectively. This analysis showed that the GC content at second position is different from the GC content at the first and third position. $\mathrm{GC} 1$ was extremely close to $\mathrm{GC} 3$, and $\mathrm{GC} 2$ was the lowest of all 3 codon positions. The average GC content of all codons was 0.503 .

Neutrality analysis is a useful way to revealing the relationship between GC12 and GC3 and then examining the mutation-selection equilibrium in shaping the CUB. In neutrality plot, if the correlation between GC12 and GC3 is statistically significant and the slope of the regression line is close to 1 , mutation bias is assumed to be the main force shaping codon usage. Conversely, selection against mutation bias can cause a narrow distribution of GC content and no correlation between GC12 and GC3 $[29,36]$. To analyze relations among the three codon positions, neutrality plots (GC12 versus GC3) were performed for $T$. saginata. It was observed that $T$. saginata genes had a wide range of GC3s ( 0.7 to 98.40) and there is a significant correlation between GC12 and GC3 $(\mathrm{r}=0.123, \mathrm{p}<0.01)$ (Figure 2), suggesting mutation and selection are probably both acting to codon usage bias in T. saginata genome. In addition, the significantly positive correlation in neutrality plots indicated intragenomic GC mutation bias affects the GC content at all codon positions in a similar manner.

\section{Relation between ENC and GC3}

To understand the relation between nucleotide composition and codon bias of $T$. saginata sequences, the values 
Table 1 Codon usage in T. saginata and T. pisiformis

\begin{tabular}{|c|c|c|c|c|c|c|c|c|c|c|c|}
\hline \multirow[b]{2}{*}{ AA } & \multirow[b]{2}{*}{ Codon } & \multicolumn{2}{|c|}{ T. saginata } & \multicolumn{2}{|c|}{ T. pisiformis } & \multirow[b]{2}{*}{ AA } & \multirow[b]{2}{*}{ Codon } & \multicolumn{2}{|c|}{ T. saginata } & \multicolumn{2}{|c|}{ T. pisiformis } \\
\hline & & $N$ & $\mathrm{RSCU}$ & $\bar{N}$ & $\mathrm{RSCU}$ & & & $N$ & RSCU & $N$ & $\mathrm{RSCU}$ \\
\hline \multirow[t]{2}{*}{ Phe } & UUU & 99671 & 0.97 & 33874 & 0.93 & Ser & UCU & 89398 & 1.11 & 26049 & 1.12 \\
\hline & UUC & 106107 & 1.03 & 39075 & 1.07 & & UCC & 97503 & 1.21 & 27379 & 1.18 \\
\hline \multirow[t]{6}{*}{ Leu } & UUA & 38856 & 0.45 & 12571 & 0.41 & & UCA & 82438 & 1.02 & 23335 & 1 \\
\hline & UUG & 97522 & 1.13 & 33937 & 1.12 & & UCG & 72259 & 0.9 & 21063 & 0.9 \\
\hline & CUU & 107259 & 1.24 & 39632 & 1.3 & Pro & $\mathrm{CCU}$ & 85183 & 1.1 & 25765 & 1.12 \\
\hline & CUC & 119595 & 1.38 & 41428 & 1.36 & & $\mathrm{CCC}$ & 79819 & 1.03 & 23072 & 1.01 \\
\hline & CUA & 52511 & 0.61 & 17904 & 0.59 & & CCA & 88760 & 1.14 & 26002 & 1.13 \\
\hline & CUG & 103594 & 1.2 & 36893 & 1.21 & & CCG & 56540 & 0.73 & 16969 & 0.74 \\
\hline \multirow[t]{3}{*}{ Ile } & AUU & 109448 & 1.3 & 41232 & 1.32 & Thr & $\mathrm{ACU}$ & 88533 & 1.13 & 29031 & 1.17 \\
\hline & AUC & 96607 & 1.15 & 36117 & 1.16 & & $\mathrm{ACC}$ & 89404 & 1.14 & 27437 & 1.11 \\
\hline & AUA & 47039 & 0.56 & 16259 & 0.52 & & ACA & 77048 & 0.98 & 23542 & 0.95 \\
\hline Met & AUG & 113948 & 1 & & & & ACG & 58709 & 0.75 & 18939 & 0.77 \\
\hline \multirow[t]{4}{*}{ Val } & GUU & 95565 & 1.12 & 35811 & 1.19 & Ala & GCU & 122134 & 1.21 & 45524 & 1.3 \\
\hline & GUC & 85970 & 1 & 29881 & 0.99 & & GCC & 111542 & 1.11 & 38284 & 1.09 \\
\hline & GUA & 45908 & 0.54 & 16165 & 0.54 & & GCA & 95661 & 0.95 & 31761 & 0.91 \\
\hline & GUG & 115101 & 1.34 & 38575 & 1.28 & & GCG & 73950 & 0.73 & 24715 & 0.7 \\
\hline \multirow[t]{2}{*}{ Tyr } & UAU & 55514 & 0.81 & 21083 & 0.81 & Cys & UGU & 55868 & 0.99 & 17870 & 0.96 \\
\hline & UAC & 81868 & 1.19 & 30723 & 1.19 & & UGC & 57522 & 1.01 & 19338 & 1.04 \\
\hline \multirow[t]{2}{*}{ His } & $C A U$ & 62791 & 0.95 & 21227 & 0.97 & Arg & CGU & 74482 & 1.33 & 26854 & 1.42 \\
\hline & CAC & 68855 & 1.05 & 22602 & 1.03 & & CGC & 67662 & 1.2 & 24579 & 1.3 \\
\hline \multirow[t]{2}{*}{ Gln } & CAA & 102281 & 0.96 & 32614 & 0.92 & & $\mathrm{CGA}$ & 67187 & 1.2 & 22254 & 1.18 \\
\hline & CAG & 110043 & 1.04 & 38283 & 1.08 & & CGG & 41070 & 0.73 & 13883 & 0.74 \\
\hline \multirow[t]{2}{*}{ Asn } & AAU & 111522 & 1.07 & 38069 & 1.05 & Ser & $A G U$ & 76215 & 0.94 & 21586 & 0.93 \\
\hline & $A A C$ & 97493 & 0.93 & 34636 & 0.95 & & AGC & 66542 & 0.82 & 20367 & 0.87 \\
\hline \multirow[t]{2}{*}{ Lys } & AAA & 115265 & 0.92 & 41472 & 0.88 & Arg & AGA & 44857 & 0.8 & 13883 & 0.74 \\
\hline & AAG & 135856 & 1.08 & 52821 & 1.12 & & AGG & 41815 & 0.74 & 13184 & 0.7 \\
\hline \multirow[t]{2}{*}{ Asp } & GAU & 147181 & 1.1 & 54522 & 1.1 & Gly & GGU & 108511 & 1.38 & 36512 & 1.36 \\
\hline & GAC & 120957 & 0.9 & 44541 & 0.9 & & GGC & 86502 & 1.1 & 30139 & 1.12 \\
\hline \multirow[t]{2}{*}{ Glu } & GAA & 152714 & 0.91 & 56449 & 0.93 & & GGA & 76898 & 0.98 & 27580 & 1.03 \\
\hline & GAG & 181548 & 1.09 & 65410 & 1.07 & & GGG & 41741 & 0.53 & 13277 & 0.49 \\
\hline
\end{tabular}

$\mathrm{N}$ : the number of codons; RSCU: Relative synonymous codon usage. The frequently used codons are displayed in bold.

of ENC were plotted against the fraction of GC at the third codon position (GC3s) (Figure 3) [28]. The ENC values of different genes ranged from 21.0 to 61, indicating that there are significant differences in codon bias among these genes. From Figure 3 it is obvious that a very considerable proportion of points lies near to the expected curve, which indicates that ENCs of most genes were close to the expected values based on their GC3s. Meanwhile, there are also some points with low ENC lying below the expected curve suggesting these genes have additional codon usage bias that is independent of GC3s. To obtain a more accurate estimate for the difference observed and expected ENC values, we calculated (ENCexp-ENCobs)/ENCexp. The frequency distributions of (ENCexp-ENCobs)/ENCexp are shown in Figure 4. Interestingly, the peak located in 0-0.05 of (ENCexp-ENCobs)/ENCexp value and most genes have -0.05-0.1 of (ENCexp-ENCobs)/ENCexp values indicating that most genes have ENCs slightly difference with expected ENC values from their GC3s. These results suggest that most genes have observed ENCs close to the expected ENC based on GC3s, though a significant number has much lower observed ENCs.

\section{Correspondence analysis}

In this thesis, we further investigate the synonymous codon usage variation among genes of $T$. saginata by correspondence analysis in RSCU. The first two axes 


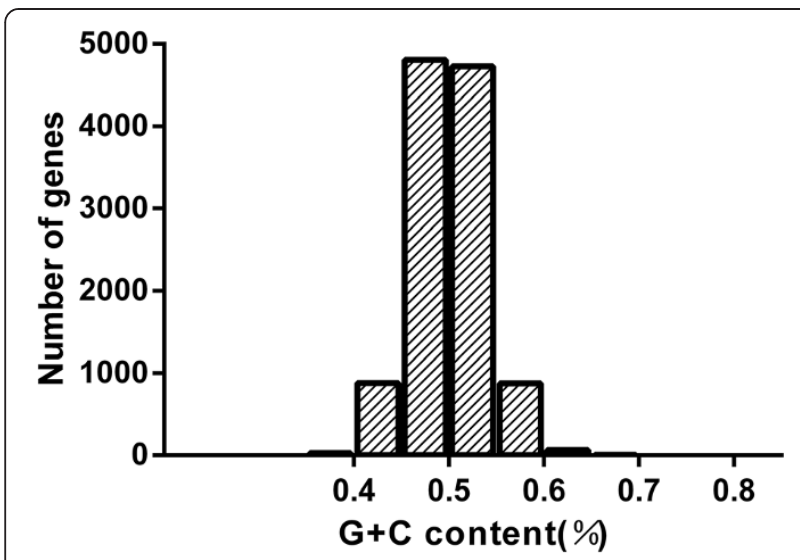

Figure 1 The distribution of $\mathrm{G}+\mathrm{C}$ contents in Taenia saginata genes.

explain low fractions of the total variation(6.6\%and $4.9 \%$, respectively), and the next two axes accounts for $4.1 \%$ and $3.3 \%$, respectively. The plot of the first and second axis of each gene is shown in Figure 5A. The distance between genes on the plot is a reflection of their diversity in RSCU. To investigate the effect of GC content of genes on codon usage bias, different GC contents of genes are color coded. The genes, GC content of which is more than or equal to $60 \%$, are plotted in green, and less than $45 \%$ is plotted in red. Blue dots indicate the genes which the GC content is between 45 and $60 \%$. In Figure 5A, the high and low GC content of genes separate along the primary axis. Correspondence analysis shows the distribution of genes in the multidimensional space, and also shows the corresponding distribution of synonymous codons (Figure $5 \mathrm{~B}$ ). Figure $5 \mathrm{~B}$ shows the separation of different bases ending codons along the two axes. The separation of codons on the first axis appears to be largely due to frequency differences in $\mathrm{G} / \mathrm{C}$ and $\mathrm{A} / \mathrm{T}$ ending codons. Further calculations revealed a significant correlation $(r=0.6573, \mathrm{P}<0.0001)$ between

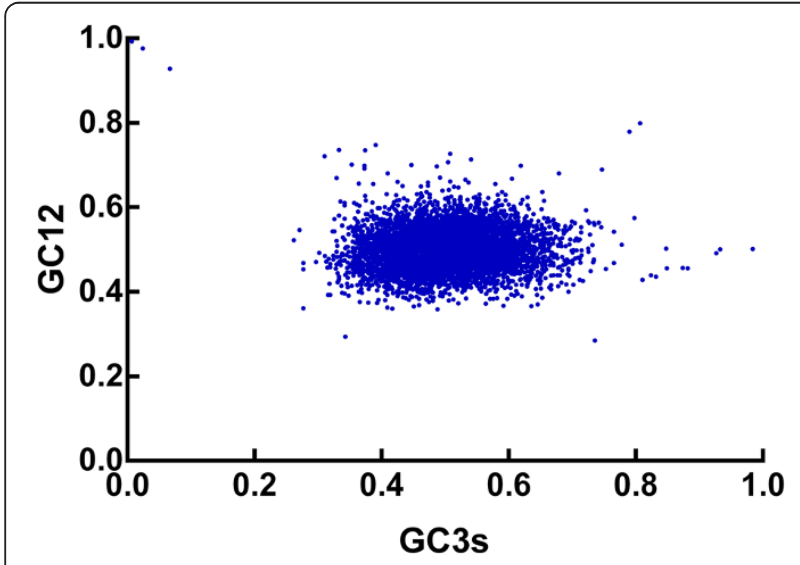

Figure 2 Neutrality plots (GC12 vs GC3s).

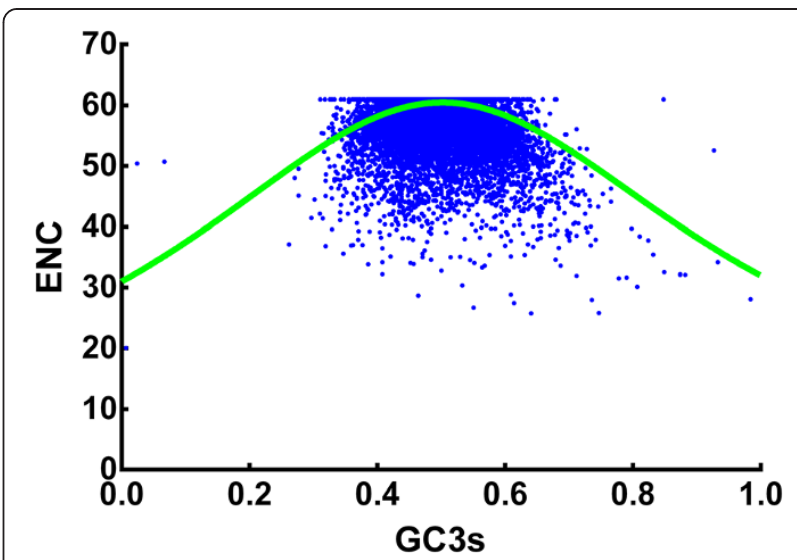

Figure 3 Distribution of effective number of codons (ENC) and GC3s of Taenia saginata genes. The solid line (shown in green) indicates the expected ENC value if the codon bias is only due to GC3s.

the GC content of individual genes and their positions on the first axis. In addition, the gene positions on axis 1 were strongly correlated with the GC3s value $(r=0.8253$, $\mathrm{P}<0.0001)$ and significantly negatively correlated with ENC $(r=-0.2322, \mathrm{P}<0.0001)$. From the above results, we found that genes with higher GC and GC3s content values and lower ENC values, which located at the left side of the first axis, indicated a stronger codon bias. This proved that the major factor influencing the codon usage bias among Taenia saginata genes was the nucleotide composition of the genes.

In order to analyze the codon usage of different kinds of gene, we selected the hydrophobic genes with gene scores $>5$, the aromatic genes with gene scores $\geq 0.15$, ribosomal genes and other genes from 11399 genes. The distribution of the four types of genes were shown in

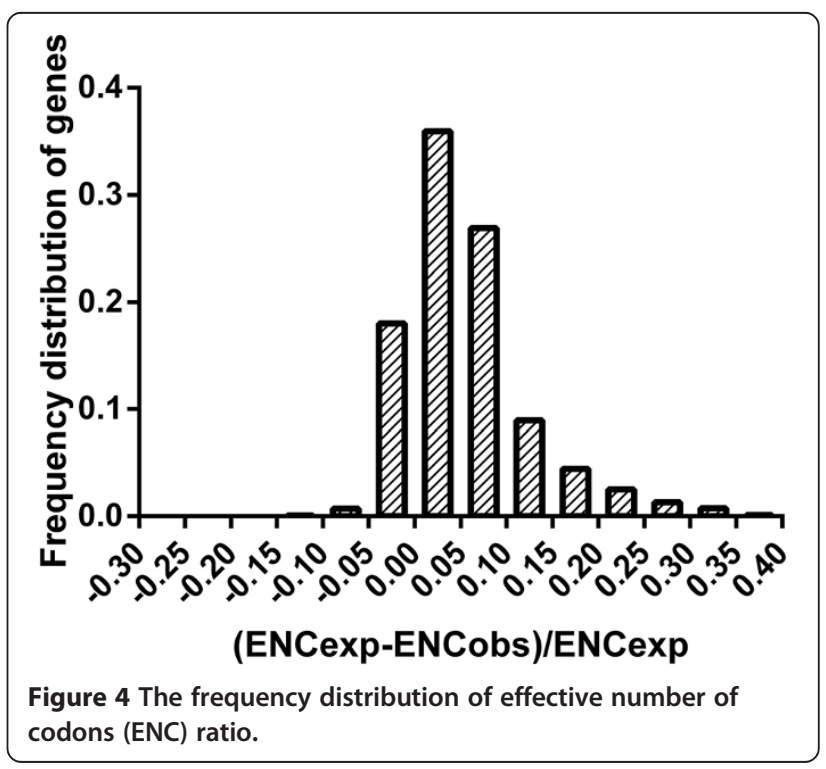



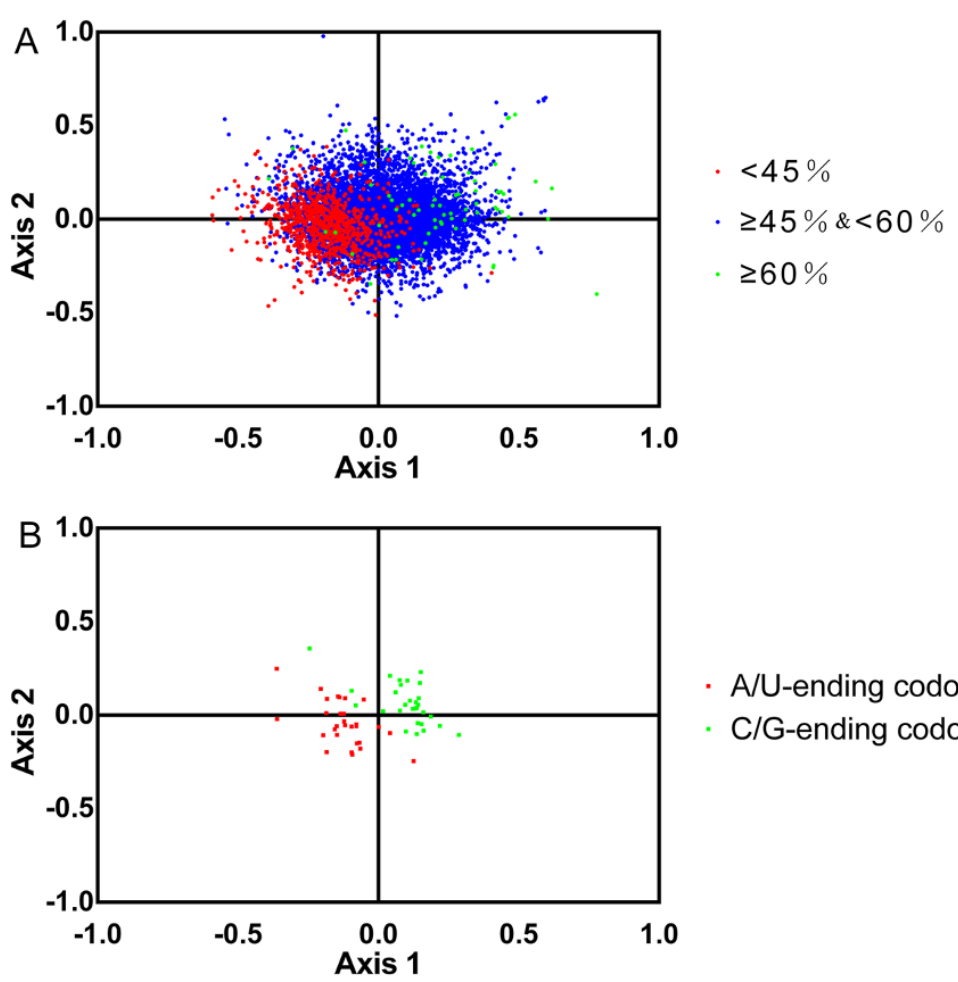

\section{- A/U-ending codons \\ C/G-ending codons}

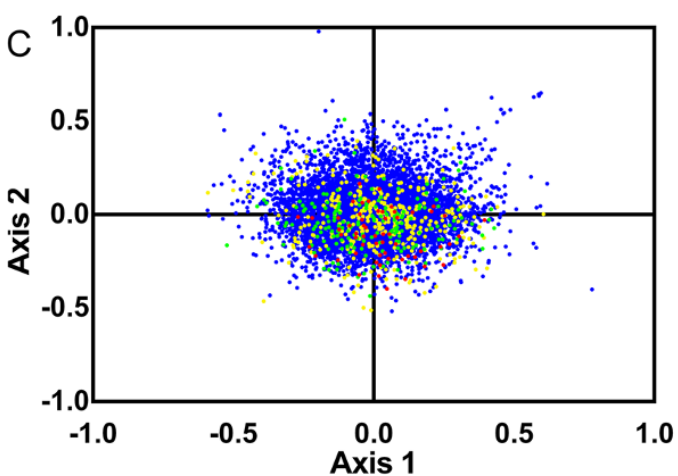

\section{Ribosomal genes} Gravy $\geq 5$

Aromo $\geq 0.15$

- Other genes

Figure $\mathbf{5}$ Correspondence analysis of relative synonymous codon usage for Taenia saginata genes. A. The distribution of genes is shown along the first and second axes. Green, blue and red dots indicate genes with $\mathrm{G}+\mathrm{C}$ content more than or equal to $60 \%$, more than or equal to $45 \%$, but less than $60 \%$ and less than $45 \%$, respectively. B. The distribution of codons on the same two axes as shown in Panel A. Codons ending with $\mathrm{A}$ and $\mathrm{T}$ are shown in red, Codons ending with $\mathrm{C}$ and $\mathrm{G}$ are shown in green. $\mathbf{C}$. Red dots, yellow dots, green triangles and blue dots indicate ribosomal genes, genes with a Aromo value more than or equal to 0.15, genes with a Gravy value higher than 5 and other genes, respectively.

Figure $5 \mathrm{C}$. We employed a multivariate analysis of variance (MANOVA) and found that there was a statistically significant difference among four types of genes in codon usage $(\mathrm{p}<0.01)$.

\section{PR-bias plot}

If mutation bias is the cause of codon bias, GC or AT should be used proportionally among the degenerate codon groups in a gene. In contrast, natural selection for codon choice would not necessarily cause proportional use of $\mathrm{G}$ and $\mathrm{C}$ (A and T) [37]. To investigate whether these biased codon choices are restricted in highly biased genes, the relation between $\mathrm{G}$ and $\mathrm{C}$ content and between $\mathrm{A}$ and $\mathrm{T}$ content in four-fold degenerate codon families were analyzed by PR2 bias plot (Figure 6). The four-codon amino acids are alanine, arginine (CGA, CGT, CGG, CGC), glycine, leucine (CTA, CTT, CTG, CTC), proline, serine (TCA, TCT, TCG, TCC), threonine, and valine. Our results showed that $\mathrm{C}$ and $\mathrm{T}$ were used more frequently than $\mathrm{G}$ and $\mathrm{A}$ in $T$. saginata. This observation indicated that both mutation bias and other factors (eg. selection) contribute to codon bias. 


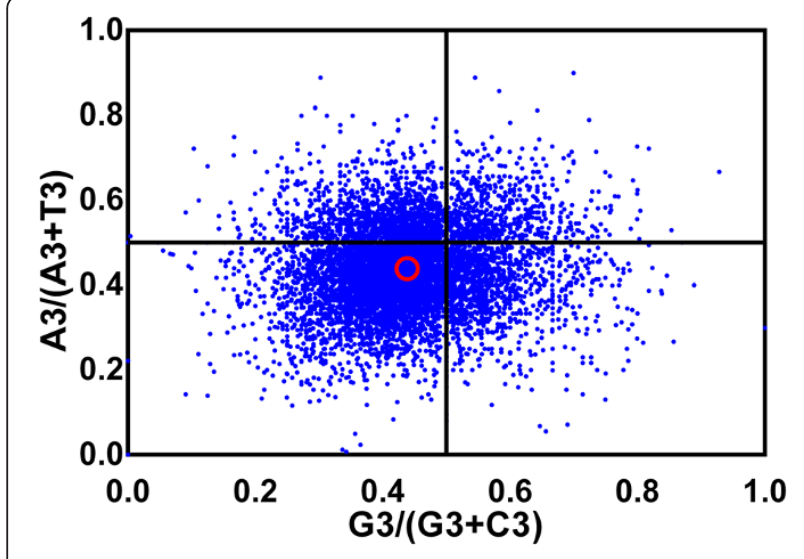

Figure 6 PR2-bias plot [A3/(A3 + T3) against G3/(G3 + C3)]. Red open circle indicate the average position for each plot. Average position is $x=0.4384 \pm 0.09663, y=0.438562 \pm 0.09486$.

Gene expression level and synonymous codon usage bias Codon adaptation index (CAI) has been extensively used as a predictor of gene expression level $[8,9]$. The set of reference sequences used for calculating CAI values in this study are genes that encode ribosomal proteins. The expression level of genes of $T$. saginata was assessed through CAI values, which varied from 0.055 to 0.952 with a mean value of 0.22 and a standard deviation of 0.03504 . It was found that there was a significantly negative correlation between the gene expression level assessed by CAI value and ENC values $(r=-0.1808$ and $\mathrm{p}<0.0001$ ) (Figure 7), and three significantly positive correlations between CAI value and GC3s, GC content and the positions of genes along axis $1(\mathrm{r}=0.2437$, 0.1009 and 0.4211 , respectively, $\mathrm{P}<0.0001$ ). The results indicated that the genes with higher expression level had a greater degree of codon usage bias and prefer the codons with $\mathrm{C}$ or $\mathrm{G}$ at the synonymous position.

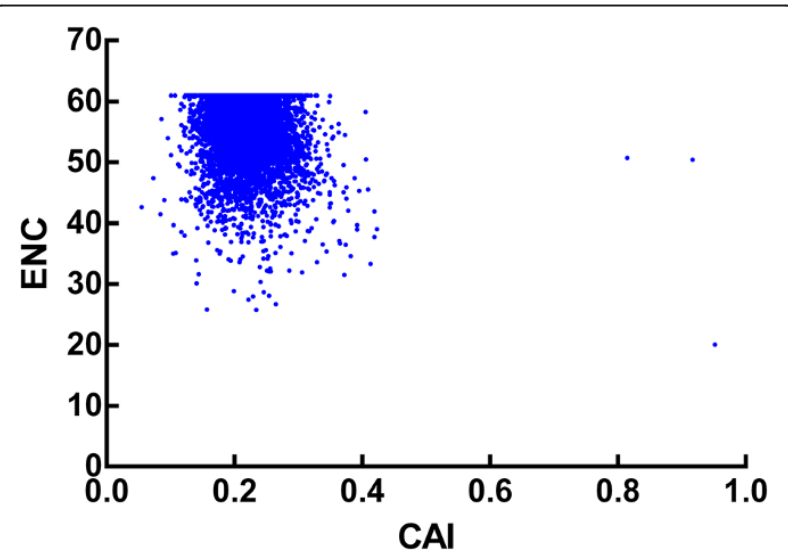

Figure 7 Plot of ENC versus gene expression level for Taenia saginata.
Protein length and synonymous codon usage bias

The results of correlation analyses between protein length and axis 1 coordinates, ENC and CAI values showed that the 3 correlation coefficients $(r=-0.1163$, 0.1433 and -0.081 , respectively, $P<0.01$ ) all significantly correlated (Figure 8), which suggested a general tendency of more biased genes with shorter length to have higher expression level.

\section{Effect of the hydrophobicity and aromaticity of encoded protein on codon bias}

Numerous studies have shown that hydrophobicity and aromaticity of encoded protein play important roles in shaping codon usage of some species. In order to investigate if the same thing is happening to $T$. saginata, we performed a correlation analysis to evaluate whether Gravy and Aromo values were related to ENC values. The correlation analyses between the hydrophobicity of each protein and ENC value showed that the correlation coefficients $(\mathrm{r}=-0.0883, \mathrm{P}<0.001)$ were significantly correlated. The aromaticity of each protein was not significantly correlated with ENC ( $r=0.0097, \mathrm{P}>0.05)$. The analysis results indicated that variation in codon usage were associated with the degree of hydrophobicity, but not with the aromatic amino acids .

\section{Optimal codons}

The average RSCU values of high/low expressed gene sample group are listed in Table 2 . Twenty-three codons were determined to be the optimal codons, which were significantly more frequent among the highly expressed genes $(\mathrm{P}<0.01)$ according to the chi-square test. Almost all of optimal codons (except GGU and CGU) ended with $\mathrm{G}$ or $\mathrm{C}$, indicating that codon usage in T. saginata was biased to $\mathrm{G}$ - or $\mathrm{C}$-ending synonymous codons.

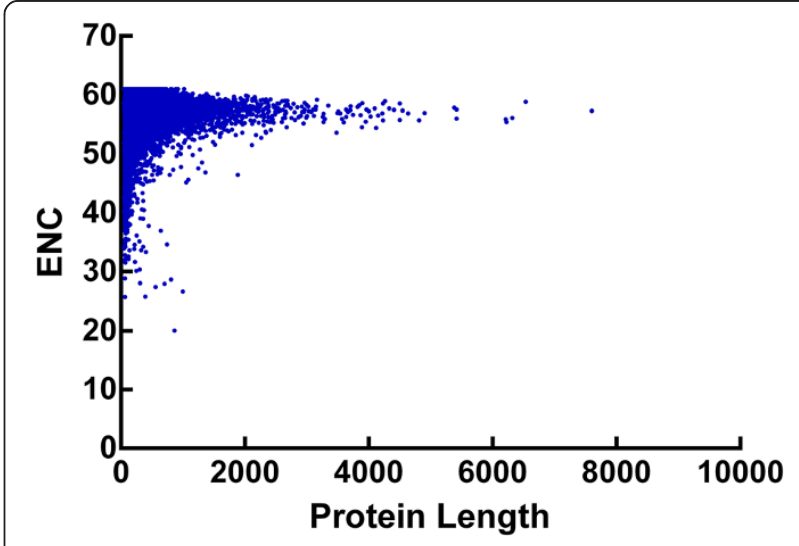

Figure 8 Plot of ENC versus protein length for Taenia saginata. 
Table 2 Translational optimal codons of $T$. saginata

\begin{tabular}{|c|c|c|c|c|c|c|c|}
\hline $\mathrm{AA}$ & Codon & High RSCU(N) & Low RSCU(N) & AA & Codon & High RSCU(N) & Low RSCU(N) \\
\hline \multirow[t]{2}{*}{ Phe } & UUU & $0.69(2341)$ & $1.23(3032)$ & Ser & UCU & $0.80(1672)$ & $1.26(2823)$ \\
\hline & $U U C^{*}$ & $1.31(4441)$ & 0.77 (1907) & & UCC* & $1.51(3157)$ & $0.87(1951)$ \\
\hline \multirow[t]{6}{*}{ Leu } & UUA & $0.20(540)$ & $0.90(1844)$ & & UCA & $0.74(1540)$ & $1.32(2967)$ \\
\hline & UUG & $0.96(2598)$ & 1.35 (2759) & & $\mathrm{UCG}^{*}$ & $1.06(2216)$ & 0.79 (1772) \\
\hline & CUU & $0.95(2570)$ & $1.34(2750)$ & & AGU & 0.85 (1770) & 1.03 (2318) \\
\hline & $\mathrm{CUC}^{*}$ & 1.97 (5332) & 0.79 (1628) & & $\mathrm{AGC}^{*}$ & $1.03(2150)$ & $0.73(1640)$ \\
\hline & CUA & 0.41 (1099) & $0.74(1516)$ & Pro & $\mathrm{CCU}$ & 0.91 (1919) & $1.21(2292)$ \\
\hline & CUG* & $1.52(4118)$ & $0.88(1804)$ & & $\mathrm{CCC}^{*}$ & 1.32 (2776) & 0.74 (1399) \\
\hline \multirow[t]{3}{*}{ \|e } & AUU & $1.02(2797)$ & $1.43(3065)$ & & CCA & $0.89(1875)$ & $1.45(2734)$ \\
\hline & $A \cup C^{*}$ & $1.61(4442)$ & $0.78(1677)$ & & CCG & $0.87(1821)$ & 0.60 (1139) \\
\hline & AUA & $0.37(1025)$ & 0.79 (1702) & Thr & $\mathrm{ACU}$ & $0.96(2294)$ & $1.21(2521)$ \\
\hline Met & AUG & $1.00(4032)$ & $1.00(3118)$ & & $\mathrm{ACC}^{*}$ & $1.49(3545)$ & $0.83(1731)$ \\
\hline \multirow[t]{4}{*}{ Val } & GUU & $0.67(1925)$ & 1.40 (2912) & & ACA & 0.70 (1678) & $1.25(2607)$ \\
\hline & GUC* & $1.14(3248)$ & $0.79(1635)$ & & ACG & $0.85(2015)$ & $0.71(1486)$ \\
\hline & GUA & $0.37(1061)$ & 0.77 (1598) & Ala & GCU & $1.00(3271)$ & $1.31(3013)$ \\
\hline & $\mathrm{GUG}^{*}$ & $1.82(5203)$ & 1.05 (2177) & & $\mathrm{GCC}^{*}$ & $1.38(4515)$ & $0.80(1840)$ \\
\hline \multirow[t]{2}{*}{ Tyr } & UAU & $0.50(1151)$ & $1.13(1851)$ & & GCA & $0.71(2315)$ & $1.28(2935)$ \\
\hline & $\cup A C^{*}$ & $1.50(3424)$ & $0.87(1414)$ & & $\mathrm{GCG}^{*}$ & 0.92 (3009) & $0.62(1417)$ \\
\hline \multirow[t]{2}{*}{ His } & $C A U$ & 0.65 (1279) & 1.23 (1936) & Cys & UGU & $0.79(1441)$ & $1.08(1673)$ \\
\hline & $C A C^{*}$ & $1.35(2642)$ & $0.77(1216)$ & & UGC* & $1.21(2188)$ & $0.92(1416)$ \\
\hline \multirow[t]{2}{*}{ Gln } & CAA & $0.71(2117)$ & 1.18 (3336) & Trp & UGG & $1.00(2015)$ & 1.00 (1293) \\
\hline & $\mathrm{CAG}^{*}$ & $1.29(3842)$ & $0.82(2309)$ & Arg & $\mathrm{CGU}^{*}$ & $1.60(2637)$ & $0.93(1267)$ \\
\hline \multirow[t]{2}{*}{ Asn } & $A A U$ & $0.79(2261)$ & 1.28 (3935) & & $\mathrm{CGC}^{*}$ & $1.88(3105)$ & $0.61(830)$ \\
\hline & $A A C^{*}$ & 1.21 (3495) & 0.72 (2199) & & CGA & 0.99 (1639) & $1.03(1414)$ \\
\hline \multirow[t]{2}{*}{ Lys } & AAA & $0.62(2293)$ & $1.10(4690)$ & & CGG & 0.71 (1176) & $0.63(858)$ \\
\hline & $\mathrm{AAG}^{*}$ & $1.38(5114)$ & $0.90(3805)$ & & AGA & $0.34(567)$ & $1.62(2220)$ \\
\hline \multirow[t]{2}{*}{ Asp } & GAU & 0.87 (3333) & $1.24(4539)$ & & AGG & $0.48(795)$ & $1.18(1612)$ \\
\hline & $G A C^{*}$ & $1.13(4308)$ & $0.76(2767)$ & Gly & $\mathrm{GGU}^{*}$ & $1.40(3673)$ & 1.18 (2195) \\
\hline \multirow[t]{3}{*}{ Glu } & GAA & $0.56(2716)$ & $1.13(6131)$ & & $\mathrm{GGC}^{*}$ & 1.46 (3819) & $0.80(1487)$ \\
\hline & $\mathrm{GAG}^{*}$ & $1.44(6946)$ & 0.87 (4759) & & GGA & $0.66(1742)$ & 1.39 (2572) \\
\hline & & & & & GGG & $0.48(1252)$ & $0.62(1158)$ \\
\hline
\end{tabular}

Comparison of codon usage frequencies between highly and lowly expressed sequences of $T$. siginata genes. AA: amino acid; N: number of codons; RSCU: Relative synonymous codon usage. Codon usage was compared using Chi squared contingency test to identify optimal codons. Asterisk denote codons that occurred significantly more often $(P<0.01)$.

\section{Discussion}

Codon usage bias is an important and complex evolutionary phenomenon, and it exists in a wide variety of organisms, from prokaryotes, to unicellular and multicellular eukaryotes. Some hypotheses are proposed to explain the origin of codon usage bias, among which neutral theory [38] and the selection-mutation-drift balance model $[27,39]$ are the most representative ones. According to neutral theory, mutations at degenerate coding positions should be selectively neutral, thus resulting in random synonymous codon choice. In the selection-mutation-drift model, codon bias is thought to be determined by a balance between mutation pressure, genetic drift, and weak selection. In other words, if a gene experiences a highly selective pressure, such as high expression, it may be inclined to stronger codon usage bias. However, in recent years, with the completion of genome projects of many organisms, the two hypotheses are not sufficient to explain codon usage anymore. Many other factors have been reported to influence CUB, including gene length [11], GC-content $[40,41]$, recombination rate $[40,42,43]$, gene expression level $[11,18,42]$, RNA structure [44-46], protein 
structure [47], intron length [48], population size [49], evolutionary age of the genes [50], environmental stress [51], the hydrophobicity and the aromaticity of the encoded proteins [52,53], and so on. In this study, the factors involved in shaping codon usage of the Taenia saginata genome at least includes gene expression level, gene compositional constraint, protein length, as well as the hydrophobicity of each protein (slightly).

Nucleotide composition could be one of the most important factors in shaping codon usage among genes and genomes. GC-rich organisms, such as Bacteria, Archea, Fungi. Triticum Aestivum, Hordium vulgare and Oryza sativa [36,54], tend to use $\mathrm{G}$ or $\mathrm{C}$ in the third position. And meanwhile, AT-rich organisms show a preference for A or $\mathrm{T}$ in third position, such as Onchocerca volvulus, Mycoplasma capricolum and Plasmodium falciparum [55-57]. The genomic G $+C$ content for $T$. saginata is $43.61 \%$. Although the genome would thus appear to be slightly $\mathrm{A}+\mathrm{T}$ rich, overall codon usage is biased toward C- and G-ending codons (Table 2), this is similar to that in Giardia lamblia [13].

Previous studies have found significant negative correlations between protein length and CUB in variety of organsims, such as yeast [58], Caenorhabditis elegans [11], Drosophila melanogaster [41], Arabidopsis thaliana [11] and Silene latifolia [59]. Similar results have also been found in T. saginata. There is an explanation proposed by Moriyama and Powell for this phenomenon: namely, if shorter proteins could perform similar functions to those of longer ones, longer proteins become energy-expensive and disadvantageous, thus the selection constraint acts to reduce the size of highly expressed genes, dominantly determines the relationship between codon bias and gene length [60].

As we know, it is difficult to quantify the expression level of genes in a differentiated multicellular eukaryote, where genes are expressed at different levels in different tissues and at different developmental stages. In the $T$. saginata genome, the expression level of an individual gene is lacking. It is known that EST counting is efficient for assessing gene expression level. Nevertheless, due to the limitation of EST numbers and rough prediction of gene expression level by counting ESTs, so we use the "Codon Adaptation Index" to evaluate the expression level of examined genes. CAI has been widely used to examine the expressivities of genes by many researchers and has now been considered as a well-accepted measure of gene expression $[8,9]$.

In this study, we identified 23 codons as the optimal codons. Most of all optimal codons in the $T$. saginata genome end with $\mathrm{G}$ or $\mathrm{C}$. This is very similar to the pattern observed in other eukaryotic genomes, such as Dictyostelium discoideum [61], D. melanogaster [62], C. elegans [18], Giardia lamblia [13] and Schizosaccharomyces pombe
[34]. The identification of optimal codons may provide useful clues for molecular genetic engineering and evolutionary studying.

\section{Conclusions}

For the first time, we have reported the pattern of codon usage bias in the $T$. saginata genome and its causative factors. Evidence suggests that the codon usage pattern in T. saginata appears to be the result of a complex equilibrium between different forces, namely mutation bias, natural selection, the GC content of genes, protein length, gene expression level and hydropathicity. Meanwhile, 23 optimal codons were identified, all of which ended with either a $\mathrm{G}$ or $\mathrm{C}$ residue, this will be useful for cloning and expression of foreign genes in the organism. Such information from this study will provide a better understanding of the characteristics of synonymous codon usage in $T$. saginata and its molecular evolution, and provide a new resource to underpin the development of urgently needed treatments and control.

\section{Competing interests}

The authors declare that they have no competing interests.

\section{Authors' contributions}

$X Y$ performed most of the data analysis work and contributed to the writing process. XY, XNL and XPC assisted with data analysis work and provided intellectual input to the work. All authors read and approved the final manuscript.

\section{Acknowledgments}

We thank several reviewers for helpful comments on the work presented here. Project support was provided by the Science Fund for Creative Research Groups of Gansu Province (Grant No. 1210RJIA006) and opening projects of National Key Laboratory of Veterinary Etiological Biology at Lanzhou Veterinary Research Institute of Chinese Academy of Agricultural Sciences (Grant Number: 201001).

Received: 11 April 2014 Accepted: 6 November 2014

Published online: 02 December 2014

\section{References}

1. Akashi H, Eyre-Walker A: Translational selection and molecular evolution. Curr Opin Genet Dev 1998, 8(6):688-693.

2. Akashi H: Gene expression and molecular evolution. Curr Opin Genet Dev 2001, 11(6):660-666.

3. Duret $L$ : Evolution of synonymous codon usage in metazoans. Curr Opin Genet Dev 2002, 12(6):640-649.

4. Hershberg R, Petrov DA: Selection on codon bias. Annu Rev Genet 2008, 42:287-299.

5. Kane JF: Effects of rare codon clusters on high-level expression of heterologous proteins in Escherichia coli. Curr Opin Biotechnol 1995, 6(5):494-500

6. Ahn I, Jeong B-J, Bae S-E, Jung J, Son HS: Genomic analysis of influenza A viruses, including avian flu (H5N1) strains. Eur J Epidemiol 2006, 21(7):511-519.

7. Zheng Y, Zhao WM, Wang H, Zhou YB, Luan Y, Qi M, Cheng YZ, Tang W, Liu J, Yu H, Yu XP, Fan YZ, Yang X: Codon usage bias in Chlamydia trachomatis and the effect of codon modification in the MOMP gene on immune responses to vaccination. Biochem Cell Biol 2007, 85(2):218-226.

8. Naya H, Romero H, Carels N, Zavala A, Musto H: Translational selection shapes codon usage in the GC-rich genome of Chlamydomonas reinhardtii. FEBS Lett 2001, 501(2):127-130 
9. Gupta S, Bhattacharyya T, Ghosh TC: Synonymous codon usage in Lactococcus lactis: mutational bias versus translational selection. J Biomol Struct Dyn 2004, 21(4):527-535.

10. Lin K, Kuang Y, Joseph JS, Kolatkar PR: Conserved codon composition of ribosomal protein coding genes in Escherichia coli, Mycobacterium tuberculosis and Saccharomyces cerevisiae: lessons from supervised machine learning in functional genomics. Nucleic Acids Res 2002, 30(11):2599-2607.

11. Duret $L$, Mouchiroud D: Expression pattern and, surprisingly, gene length shape codon usage in Caenorhabditis, Drosophila, and Arabidopsis. Proc Natl Acad Sci U S A 1999, 96(8):4482-4487.

12. Kliman RM, Irving N, Santiago M: Selection conflicts, gene expression, and codon usage trends in yeast. J Mol Evol 2003, 57(1):98-109.

13. Lafay B, Sharp PM: Synonymous codon usage variation among Giardia lamblia genes and isolates. Mol Biol Evol 1999, 16(11):1484-1495.

14. Ghosh TC, Gupta SK, Majumdar S: Studies on codon usage in Entamoeba histolytica. Int J Parasitol 2000, 30(6):715-722.

15. Wright F, Bibb MJ: Codon usage in the G + C-rich Streptomyces genome. Gene 1992, 113(1):55-65.

16. Mclnerney JO: Replicational and transcriptional selection on codon usage in Borrelia burgdorferi. Proc Natl Acad Sci U S A 1998, 95(18):10698-10703.

17. Sharp PM, Cowe E: Synonymous codon usage in Saccharomyces cerevisiae. Yeast 1991, 7(7):657-678.

18. Stenico M, Lloyd AT, Sharp PM: Codon usage in Caenorhabditis elegans: delineation of translational selection and mutational biases. Nucleic Acids Res 1994, 22(13):2437-2446

19. Wanzala W, Onyango-Abuje JA, Kang'ethe EK, Zessin KH, Kyule NM, Baumann MP, Ochanda H, Harrison LJ: Control of Taenia saginata by post-mortem examination of carcasses. Afr Health Sci 2003, 3(2):68-76.

20. Dorny P, Vercammen F, Brandt J, Vansteenkiste W, Berkvens D, Geerts S: Sero-epidemiological study of Taenia saginata cysticercosis in Belgian cattle. Vet Parasitol 2000, 88(1):43-49.

21. Lightowlers MW, Rolfe R, Gauci CG: Taenia saginata: Vaccination against Cysticercosis in Cattle with Recombinant Oncosphere Antigens. Exp Parasitol 1996, 84(3):330-338.

22. Matuchansky C, Lenormand $Y$ : Images in clinical medicine. Taenia saginata N Engl J Med 1999, 341(23):1737.

23. Lees W, Nightingale J, Brown D, Scandrett B, Gajadhar A: Outbreak of Cysticercus bovis (Taenia saginata) in feedlot cattle in Alberta. Can Vet $J$ 2002, 43(3):227-228.

24. Altschul SF, Madden TL, Schaffer AA, Zhang J, Zhang Z, Miller W, Lipman DJ: Gapped BLAST and PSI-BLAST: a new generation of protein database search programs. Nucleic Acids Res 1997, 25(17):3389-3402

25. Journet EP, van Tuinen D, Gouzy J, Crespeau H, Carreau V, Farmer MJ, Niebel A, Schiex T, Jaillon O, Chatagnier O, Godiard L, Micheli F, Kahn D, Gianinazzi-Pearson V, Gamas P: Exploring root symbiotic programs in the model legume Medicago truncatula using EST analysis. Nucleic Acids Res 2002, 30(24):5579-5592.

26. Fukunishi $Y$, Hayashizaki $Y$ : Amino acid translation program for full-length cDNA sequences with frameshift errors. Physiol Genomics 2001, 5(2):81-87.

27. Sharp PM, Li W-H: An evolutionary perspective on synonymous codon usage in unicellular organisms. J Mol Evol 1986, 24(1-2):28-38.

28. Wright F: The 'effective number of codons' used in a gene. Gene 1990, 87(1):23-29.

29. Sueoka N: Directional mutation pressure and neutral molecular evolution. Proc Natl Acad Sci U S A 1988, 85(8):2653-2657.

30. Sharp PM, Li W-H: The codon adaptation index-a measure of directional synonymous codon usage bias, and its potential applications. Nucleic Acids Res 1987, 15(3):1281-1295.

31. Wang H-C, Hickey DA: Rapid divergence of codon usage patterns within the rice genome. BMC Evol Biol 2007, 7(Suppl 1):S6.

32. Liu Q, Feng $Y$, Zhao $X$, Dong $H$, Xue Q: Synonymous codon usage bias in Oryza sativa. Plant Sci 2004, 167(1):101-105.

33. Liu Q: Analysis of codon usage pattern in the radioresistant bacterium Deinococcus radiodurans. Biosystems 2006, 85(2):99-106.

34. Sharp PM, Cowe E, Higgins DG, Shields DC, Wolfe KH, Wright F: Codon usage patterns in Escherichia coli, Bacillus subtilis, Saccharomyces cerevisiae, Schizosaccharomyces pombe, Drosophila melanogaster and
Homo sapiens; a review of the considerable within-species diversity. Nucleic Acids Res 1988, 16(17):8207-8211.

35. Chen L, Liu T, Yang D, Nong X, Xie Y, Fu Y, Wu X, Huang X, Gu X, Wang S, Peng $X$, Yang G: Analysis of codon usage patterns in Taenia pisiformis through annotated transcriptome data. Biochem Biophys Res Commun 2013, 430(4):1344-1348.

36. Kawabe A, Miyashita NT: Patterns of codon usage bias in three dicot and four monocot plant species. Genes Genet Syst 2003, 78(5):343-352.

37. Sueoka N, Kawanishi Y: DNA G+ C content of the third codon position and codon usage biases of human genes. Gene 2000, 261(1):53-62

38. Nakamura Y, Gojobori T, Ikemura T: Codon usage tabulated from the international DNA sequence databases. Nucleic Acids Res 1997, 25(1):244-245.

39. Bulmer $\mathrm{M}$ : Are codon usage patterns in unicellular organisms determined by selection-mutation balance? J Evol Biol 1988, 1(1):15-26.

40. Comeron JM, Kreitman M, Aguade M: Natural selection on synonymous sites is correlated with gene length and recombination in Drosophila. Genetics 1999, 151(1):239-249.

41. Marais $G$, Mouchiroud D, Duret $L$ : Does recombination improve selection on codon usage? Lessons from nematode and fly complete genomes. Proc Natl Acad Sci U S A 2001, 98(10):5688-5692.

42. Hey J, Kliman RM: Interactions between natural selection, recombination and gene density in the genes of Drosophila. Genetics 2002, 160(2):595-608.

43. Kliman RM, Hey J: Hill-Robertson interference in Drosophila melanogaster: reply to Marais, Mouchiroud and Duret. Genet Res 2003, 81(2):89-90.

44. Hartl DL, Moriyama EN, Sawyer SA: Selection intensity for codon bias Genetics 1994, 138(1):227-234

45. Chen Y, Carlini DB, Baines JF, Parsch J, Braverman JM, Tanda S, Stephan W: RNA secondary structure and compensatory evolution. Genes Genet Syst 1999, 74(6):271-286.

46. Carlini DB, Chen Y, Stephan W: The relationship between third-codon position nucleotide content, codon bias, mRNA secondary structure and gene expression in the drosophilid alcohol dehydrogenase genes Adh and Adhr. Genetics 2001, 159(2):623-633.

47. Oresic M, Dehn M, Korenblum D, Shalloway D: Tracing specific synonymous codon-secondary structure correlations through evolution. $\mathrm{J}$ Mol Evol 2003, 56(4):473-484.

48. Vinogradov AE: Intron length and codon usage. J Mol Evol 2001, 52(1):2-5

49. Berg OG: Selection intensity for codon bias and the effective population size of Escherichia coli. Genetics 1996, 142(4):1379-1382.

50. Prat $Y$, Fromer M, Linial N, Linial M: Codon usage is associated with the evolutionary age of genes in metazoan genomes. BMC Evol Biol 2009, 9:285

51. Goodarzi H, Torabi N, Najafabadi HS, Archetti M: Amino acid and codon usage profiles: adaptive changes in the frequency of amino acids and codons. Gene 2008, 407(1-2):30-41.

52. Romero $\mathrm{H}$, Zavala A, Musto $\mathrm{H}$ : Codon usage in Chlamydia trachomatis is the result of strand-specific mutational biases and a complex pattern of selective forces. Nucleic Acids Res 2000, 28(10):2084-2090

53. Rispe C, Delmotte F, van Ham RC, Moya A: Mutational and selective pressures on codon and amino acid usage in Buchnera, endosymbiotic bacteria of aphids. Genome Res 2004, 14(1):44-53.

54. Hershberg R, Petrov DA: General rules for optimal codon choice. PLOS Genet 2009, 5(7):e1000556.

55. Saul A, Battistutta D: Codon usage in Plasmodium falciparum. Mol Biochem Parasitol 1988, 27(1):35-42.

56. Milhon JL, Tracy JW: Updated codon usage in Schistosoma. Exp Parasitol 1995, 80(2):353-356.

57. Muto A, Yamao F, Osawa S: The genome of Mycoplasma capricolum. Prog Nucleic Acid Res Mol Biol 1987, 34:29-58.

58. Ingvarsson PK: Gene expression and protein length influence codon usage and rates of sequence evolution in Populus tremula. Mol Biol Evol 2007, 24(3):836-844.

59. Qiu S, Bergero R, Zeng K, Charlesworth D: Patterns of codon usage bias in Silene latifolia. Mol Biol Evol 2011, 28(1):771-780. 
60. Moriyama EN, Powell JR: Codon usage bias and tRNA abundance in Drosophila. J Mol Evol 1997, 45(5):514-523.

61. Sharp PM, Li W-H: On the rate of DNA sequence evolution in Drosophila. J Mol Evol 1989, 28(5):398-402

62. Shields DC, Sharp PM, Higgins DG, Wright F: " Silent" sites in Drosophila genes are not neutral: evidence of selection among synonymous codons. Mol Biol Evol 1988, 5(6):704-716.

doi:10.1186/s13071-014-0527-1

Cite this article as: Yang et al:: Analysis of codon usage pattern in

Taenia saginata based on a transcriptome dataset. Parasites \& Vectors 2014 7:527.

\section{Submit your next manuscript to BioMed Central and take full advantage of:}

- Convenient online submission

- Thorough peer review

- No space constraints or color figure charges

- Immediate publication on acceptance

- Inclusion in PubMed, CAS, Scopus and Google Scholar

- Research which is freely available for redistribution 